Revista Iberoamericana. Vol. LXIII, Núm. 180, Julio-Setiembre 1997; 459-464

\title{
"LA NOCHE BOCA ARRIBA" COMO ENCRUCIJADA LITERARIA
}

\author{
POR \\ José AmícolA \\ Universidad Nacional de La Plata
}

Ibant obscuri sola sub nocte per umbras

Virgilio, Eneida.

En su hermosa dedicatoria a Lugones en el prólogo a El hacedor (1960), Borges exorciza la memoria de su padre literario, colocándolo en la línea prestigiosa de Virgilio y Milton a través del uso del procedimiento de la "hipálage" (del griego: "intercambio"), que allí culmina por asociación en la idea de pasaje ("pero mañana yo también habré muerto y se confundirán nuestros tiempos y la cronología se perderá en un orbe de símbolos ...", 779). ${ }^{1}$ En la década del 50 Julio Cortázar buscaba, por su parte, afianzarse dentro del sistema literario mediante la imitación de los modelos prestigiosos, pero, al mismo tiempo, quería encontrar una salida propia a cada uno de los conflictos que le suscitaba la escritura. Si los títulos de sus cuentos que indican el encuentro con el público argentino como "Casa tomada" instauran la marca de la sencillez de su maestro, al mismo tiempo, se dan los que anuncian mediante atributos artísticos el conflicto de lo inquietante. Un autor determina con sus primeras obras, por cierto, una inserción en un sistema que posee ya determinados principios de construcción. Será la nueva personalidad la que habrá de llevar a la condición de "Dominante" en su propia obra algunos de los factores constructivos que antes aparecían al borde del campo artístico. En efecto, "La noche boca arriba"(1956), el cuento que nos ocupa, continúa el gesto borgeano iniciado con "Casa tomada", pero lo lleva un paso más adelante al poner en escena lo que los formalistas rusos denominaron la exhibición del procedimiento (o artificio). El sintagma conversacional "boca arriba" de valencia humana aparece, entonces, soldado a un término de la naturaleza ("la noche"). La sintaxis refuerza el recurso al poner en contacto los dos sustantivos ("noche"-"boca") gracias a una síntesis entre la construcción nominal y la de valor adverbial; a nivel fónico ello se ve reforzado por la secuencia "ao e oa(aia)". La hipálage del título exhibe así ya, en rigor, lo que la figura del cuento instala dentro del texto de la obra mediante la idea de "pasaje o intercambio". De ese modo el universo por el que

\footnotetext{
' Las citas de este texto de Borges y de su cuento "El Sur" provienen de Obras completas 1923-1972.

2 "Casa tomada" tiene el mérito de ser un primer texto y, por lo tanto, poseer el gesto inaugural con el que su autor entra a la palestra. No es un dato menor el hecho de que Borges haya elegido este relato para su publicación en la revista Anales de Buenos Aires, 11 (nov.1946).
} 
entramos al relato reproduce en escala reducida el macrouniverso del texto que también nos lleva de la mano por la imperceptibilidad del deslizamiento de los planos puestos en juego. El principio de deslizamiento de planos había aparecido ya, en efecto, como principio constructivo tangencial en un cuento de Borges como "Tlön, Uqbar, Orbis Tertius"; sería tarea de Cortázar catapultarlo a factor Dominante. Objetivo de nuestro análisis es demostrar la función que este principio desempeña en el relato.

Cuando Laurence Sterne colocó en su novela The Life and Opinions of Tristram Shandy (1759-67) un dibujo espiralado para esquematizar la libertad de su discurso, tuvo conciencia de por dónde pasaba el conflicto de su época. Con ese diagrama Sterne construía de modo icónico el principio dominante de su obra, la Digresión, como declaración de guerra contra la honda motivación del argumento en la novela que había instituido Samuel Richardson en el panorama literario inglés. Cuando Borges comenzaba su artículo titulado "Cuando la ficción vive en la ficción" (publicado en "El Hogar" el 2 de junio de 1939), del siguiente modo: "Debo mi primera noción del problema del infinito a una gran lata de bizcochos que dio misterio y vértigo a mi niñez. En el costado de ese objeto anormal había una escena japonesa; no recuerdo los niños o los guerreros que la formaban, pero sí que en un ángulo de esa imagen la misma lata de bizcochos reaparecía con la misma figura y en ella la misma figura, y así (a lo menos, en potencia) infinitamente ..." (Textos cautivos 325), este autor estaba adelantando el descubrimiento de su principio constructivo dominante. André Gide lo había llamado ya mise-en-abyme y, aunque provenía del manierismo, iba a vivir una singular reaparición en nuestro siglo como el aparato que constituye "El Aleph". A partir de esta idea clave se erigen, en nuestra opinión, la mayor parte de los relatos borgeanos. Solamente que Borges no exhibiría como Sterne sus procedimientos constructivos.

Para Cortázar, entre tanto, el recurso literario característico de Borges presentaba un evidente atractivo, aunque no podía, sin embargo, aceptarlo sin más, en cuanto ese procedimiento evidenciaba una simetría en la repetición al infinito que no ocultaba su origen en una raigambre especulativa. En la idea borgeana estaba la infracción al marco que contenía el espejo, pero por ello no desaparecía justamente la importancia del corte en recuadro que sólo la mente ( $\mathrm{y}$, potencialmente, la percepción sensorial) podía franquear. Cortázar coincidiría con Borges en la aseveración de éste último (en el mismo artículo de "El Hogar") en que ese recurso aplicado a la literatura hacía que la realidad nos pareciera irreal, pero, en cambio, para llegar a casi los mismos resultados buscaría su propio procedimiento: la figura geométrica determinada por Möbius (1790-1868) como superficie continua infinita:

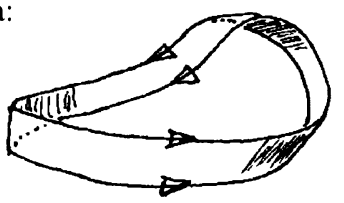

Con esta representación icónica para su obra Cortázar se alejaba, por un lado, de los moldes borgeanos (el sofisma de Zenón, el espejo del cuadro de Velázquez - todas formas, quizás, de mise-en-abyme), para acercarse, por otro lado, al espíritu de la 
modernidad. Ahora de lo que se trata es de una concepción que tiene más que ver con el espíritu de los pasajes de Walter Benjamin, con los niveles de conciencia de Freud o, inclusive, con el sentimiento de la angustia respirada por los héroes de Sartre. La cinta de Möbius habría de poner en escena no la nitidez de las fronteras, sino, por el contrario, la peligrosa fusión de bordes de territorios no reflejados de modo especular, sino presentados diametralmente contrapuestos - - en rigor, un desarrollo de la figura retórica de la hipálage. Esta condición de diferencia de los planos enfrentados ejerce, por lo tanto, un tour-de-force en la fusión de lo perceptible como distinto. La figura geométrica ahora dominante en Cortázar se manifiesta también en cuentos como "Continuidad de los parques", "El otro cielo" o "Todos los fuegos el fuego" hasta llegar a la franca exhibición del título del cuento de "Queremos tanto a Glenda" (1981): "Anillo de Moebius [sic]".3 Cortázar funda con el nuevo procedimiento un nuevo espacio para su literatura que deja de lado toda simetría y sentido de unidad de perspectiva para internarse en la comprensión de su época a través de los iconos que ahora la representan. El nuevo principio no sólo hace menos real la realidad, sino que resemantiza la inseguridad del hombre erguido sobre una cinta inasible - y en movimiento- en la época de la "reproduc(t)ibilidad técnica". "La noche boca arriba" se construye, entonces, sobre una intertextualidad borgeana que abarca no sólo la hipálage, sino también la renovación del principio constructivo dominante. Pero hay algo más. Este cuento responde como "El Sur" de Borges a un acontecimiento autobiográfico que actúa como disparador creativo. Borges y Cortázar toman como inspiración para la narración un accidente en sus vidas que los lleva al descubrimiento de otros planos de la conciencia y a la tarea posterior de aprovechar literariamente las pautas que les ha brindado el delirio durante la postración. La idea que está en la base de "La noche boca arriba" parecería provenir de una experiencia vital común con Borges: el accidente y la convalecencia. El cuento de Cortázar parecería, además desarrollar la siguiente frase de "El Sur": "La fiebre lo gastó y las ilustraciones de las Mil y Una NOCHES sirvieron para decorar pesadillas" (525). También en el cuento de Borges el protagonista se siente feliz de hallarse con vida en la clínica, también la clínica significa allí el territorio seguro: "No hubieran permitido en el sanatorio que me pasaran estas cosas" (529). Con la utilización de estos materiales originarios de la serie literaria Cortázar muestra, al mismo tiempo, sumisión y rebeldía. Contra la saturación de los indicios geográficos concretos de "El Sur", el cuento que nos ocupa degrada al estanciero Juan Dahlmann a la condición de oficinista sin nombre de una ciudad anónima con edificios ministeriales y una Calle Central, cuyo medio de locomoción es ya no el vagón de primera clase en la tradición literaria de 1900, sino la motocicleta del Hombre Común de la Calle - con la que se mueve también a gran velocidad el Antihéroe de las Vanguardias. Ahora bien, los materiales similares son elaborados por los dos grandes autores argentinos de acuerdo a los dos principios diferentes antes mencionados. La frase clave para esta comprensión de "El Sur" es breve y concisa como todo lo que caracteriza a Borges: "A la realidad le gustan las simetrías y los leves anacronismos" (526). En la obra de Cortázar el texto se desliza, en cambio, con un juego de fusión de dos planos que

\footnotetext{
${ }^{3}$ Adolfo Prieto analiza los procedimientos que en las obras de Cortázar "buscan romper el sentido unívoco del relato", pero sin llegar a la idea que aquí se sostiene (168).
} 
pasa de la atmósfera impregnada de medicamentos de la clínica a las escenas de huida en la selva, donde el protagonista es un "moteca"s que huele la caza de hombres llevada a cabo por los sacerdotes aztecas en busca de víctimas propiciatorias: el otro plano remite a lo exótico y a las raíces de América, que aparece contrastado con la falta de fijación geográfica de lo que se suponía la realidad. La organización del relato va despeñándose por abismos cada vez con mayor rapidez en su ritmo de cambios de planos hacia el final sorpresivo. Lo que ha importado a la instancia narradora es subrayar, ahora, la sutura de los cambios en modo creciente. El cuarto y último efecto de pasaje sigue de cerca al protagonista que se transforma ante nuestros ojos con esta frase:

Hizo un último esfuerzo, con la mano sana esbozó un gesto hacia la botella de agua, no llegó a tomarla, sus dedos se cerraron en un vacío otra vez negro, y el pasadizo seguía interminable, $[\ldots]$ y él boca arriba gimió apagadamente porque el techo iba a acabarse, subía, abriéndose como una boca de sombra, y los acólitos se enderezaban y de la altura una luna menguante le cayó en la cara donde los ojos no querían verla, desesperadamente se cerraban y abrían buscando pasar al otro lado, descubrir de nuevo el cielo raso protector de la sala (Final de juego, 178-179, énfasis mío).

El texto instaura así dentro de la forma lo que surge de su contenido en una singular interrelación que deja de lado el enmarque borgeano; al mismo tiempo establece un desvío que desarrolla la mirada extrañada sobre los objetos ("insecto de metal que zumbaba": la motocicleta) de un individuo en una situación de doble plano en la que se revela el Miedo como condición humana eterna. Este miedo aparece ahora elaborado, a diferencia del texto de Borges, como una lucha librada entre la conciencia y la percepción del individuo a la mejor manera del surrealismo y el existencialismo: el Hombre sin su amuleto (es decir, sin dioses) ha quedado en soledad y angustia. Del cuento borgeano ha quedado la fatalidad y el deseo de la instancia narrativa de crearse una tradición, así como el tema de la Muerte vista como la muerte propia. Pero si Juan Dahlmann muere por respetar un código ajeno, en el cruce de civilización y primitivismo (en la oposición Libro-Vida, así como a nivel de clases sociales), el protagonista de Cortázar se halla de tal modo dentro de su propio código (en la muerte que lo amenaza) que todo el texto no hace otra cosa que relativizar la perspectiva cultural en la dicotomía "cultura-natura". Ahora la vida vegetativa de la convalecencia se acerca a la dimensión Naturaleza, mientras que el plano de lo primitivo se revela como el de la Cultura, otra Cultura. Esta sería una de las torsiones de la cinta sobre la que se desliza el texto. La representación de la Otredad que tenemos ante nosotros lleva consigo, al mismo tiempo, un juego de desplazamiento del punto de vista que acompaña estrechamente la división psíquica del protagonista. Por otro lado, se instala en el texto la desconfianza frente a los datos de la conciencia y de lo que se llama Realidad, colaborando a la desestabilización del Sujeto (como escribía el mismo Cortázar en "Teoría del túnel" 1947: "después de descubrir que la realidad continúa desconocida, se pregunta si su experiencia gnoseológica no será una contraparte igualmente falsa, igualmente mal conocida" (Cortázar, Obra crítica 122). Y, en este sentido, "La noche boca arriba" es un

\footnotetext{
${ }^{4}$ Cortázar, tan afecto a los neologismos, consiguió engañar a muchos eruditos con su "moteca", que, en el fondo, juega con la idea de un "azteca en moto(-cicleta)".
} 
cuento paradigmático de la segunda mitad de nuestro siglo en el que se habla de una pérdida (o quizás mejor descentramiento) del Sujeto heredado de la filosofía cartesiana. Este acontecimiento está aquí íntimamente ligado al rol que le cabe a lo fantástico en la obra de Cortázar, pero este autor no llega a ello como su maestro (por la desconfianza de que la escritura representara la vida). ${ }^{5}$ Quizás la esencia de esa encrucijada artística esté en la frase del cuento que nos ocupa, cuando alli se afirma que: "las cosas tenían un relieve como de gemelos de teatro, eran reales y dulces y a la vez ligeramente repugnantes" (173). La agudización del recurso del detalle en cada uno de los dos planos presentados en el relato va trabajando en el texto la duda acerca de la Realidad. Por ello la precisión de los elementos de esa Otra cultura, como el puñal de piedra, la plegaria del maíz y el teocalli, así como la "caza florida", preanunciada en el epígrafe, no hace otra cosa que mandar la flecha hacia el blanco que es el final definitivo donde lo inquietante se percibe ya como el Horror. Así "el puñal" venido de la literatura gauchesca - a través de "El Sur" borgeano- se ve transformado en el "puñal de piedra", connotado ahora con la marca cultural de lo distinto - y de lo mismo - por el efecto de la cinta de Möbius. No parece ajeno a este recurso tampoco la utilización de la figura de la duplicación de la personalidad que ha sido señalada con perspicacia por Mónica Tamborenea. Para esta autora existe una "doble circulación" en los textos de Cortázar y la obsesión visual que caracterizaría a sus personajes lleva consigo la búsqueda (freudiana) del deseo y de allí la amenaza de lo siniestro que culmina en ceguera, en castración y muerte en un proceso de impulso determinista (44). Pero, además, el texto que nos ocupa manifiesta una torsión de la cinta discursiva que se produce en la identificación, pues el poder se vuelve en contra de quien lo detenta y el victimario se escinde en su víctima, como muestra este esquema:

HOMBRE-MÁQUINA

\section{(LA TÉCNICA COMO SU IDEOLOGÍA) MUJER QUE CRUZA LA CALLE}

\section{AZTECAS}

(LA RELIGIÓN COMO SU IDEOLOGÍA)

"MOTECA"

Por un ritual similar a una castración el "moteca" aparece privado del discurso de poder (masculino) y es asimilado a la pasividad de la MUJER del primer plano.

Es sabido que, desde sus comienzos como escritor, Cortázar entra al campo literario argentino, construido por aquella época como un coto privado, con la lanza de un caballero

\footnotetext{
${ }^{5}$ Por una cuestión de economía expositiva no se entrará aquí en el debate acerca de la tipología de lo fantástico. Luis Gregorich, por su parte, (Jitrik 126) ubica el cuento que nos ocupa entre las más conspicuas representaciones del género, cuando escribe: "lo fantástico se da en Cortázar como contraste entre un idioma pulidamente objetivo, sobrio, popular a ratos, sabroso, y una visión del mundo como fractura, como dualidad e incomunicación fundamental" (126). Véase también entrevista a Cortázar de Soriano/Colominas. La irrupción de lo fantástico en el mundo desencantado de nuestra época es visto justamente por Fredric Jameson como síntoma de este momento histórico cuando escribe: "When, at the end of the nineteenth century, the search for secular equivalents [the older magical content] seems exhausted, the characteristic indirection of a nascent modernism, from Kafka to Cortázar, circumscribes the place of the fantastic as a determinate, marked absence at the hart of the secular".
} 
en ristre para horadar un túnel en el sistema literario. Así entroniza las estridencias de Artaud en sus comentarios sobre ese autor en el centro del silencio camarístico de la revista de Victoria Ocampo o, por otra parte, sale a defender la novela anticanónica Adán Buenosayres, de Leopoldo Marechal en la revista irónicamente titulada "Realidad" (1949). En este contexto debe entenderse, pues, "La noche boca arriba", al mismo tiempo, como una poética del autor. Cortázar busca, en efecto, diferenciarse del realismo instaurado en las letras argentinas de la líneas de Larreta, Lynch, Gálvez o Barletta, sin dejar de lado sus concepciones acerca del género cuentístico aprendido en Poe o Quiroga. Al mismo tiempo, debe encontrar un punto de independencia de sus maestros más inmediatos como Borges (o Arlt). En este camino de búsqueda es el final de "La noche boca arriba" el que representa un viraje como desautomatización de la norma literaria. Si un texto tiene, por cierto, la virtud de hacernos creer que no existe nada fuera de él y convencernos de lo absoluto de su autonomía, también es evidente que ese texto indica a través del subtexto en qué medida el espacio ganado para el nuevo imaginario simbólico puede ser considerado una reacción. Independizándose del realismo, Cortázar gana otro espacio también, por un efecto de "carambolage", y se aleja de los antirrealistas del sistema, como el propio Borges. Por ello puede decirse que Cortázar deconstruye en el cuento analizado tanto la narración del tipo "rollo chino" como el aparato borgeano, al extender la duda perceptiva a toda la cotidianeidad y al desestabilizar así la condición estática del punto de vista, como sucede también en "Axolotl", ${ }^{6}$ donde según A. M. Barrenechea se introduce lo fantástico a nivel de la semántica global del texto. Entre el final de "El Sur" que dice lacónicamente: "Dahlmann empuña con firmeza el cuchillo, que acaso no sabrá manejar, y sale a la llanura" (530), y el final de "La noche boca arriba" existe un universo de diferencia que tiene que ver con el mismo principio que Walter Benjamin descubrió en la obra de Proust, al analizarla como cinta de Möbius. Con Benjamin (así como con el surrealismo francés) Cortázar implanta en el imaginario argentino la veneración de los pasajes (o galerías) como territorio de fusión desde la primera edición de su libro Todos los fuegos el fuego. ${ }^{7}$ El desgarramiento del mundo aparecería también

\footnotetext{
${ }^{6}$ Altamirano/Sarlo vinculan este cuento con el que nos ocupa indicando en ambos una canibalización y una mostración de la forma barroquizante para el plano americano por oposición a la simplicidad y univocidad del primer plano, que por un viraje ideológico se presenta como no verdadero. Para estos autores el principio constructivo de ambos relatos sería lo real-maravilloso como ideologema irracional. Lo fantástico lo sería, además, sólo para el lector y no para los seres que intervienen en el relato. Aquí agregaríamos, por nuestra parte, que la cinta de Möbius es visible sólo desde el exterior a ella misma. En cuanto a la aparición del territorio mexicano en los dos cuentos de Cortázar, parece más verificable que la influencia de Carpentier la relación con el sentimiento mágico que proviene de las alegorías The Plumed Serpent, de D. H.Lawrence, y Under the Volcano, de M. Lowry. Aunque Cortázar ya no necesita confrontarse con la gauchesca como le sucedía a Borges, también "se inventa una tradición": la americanidad (que siguiendo a Jameson obraría aquí como el inconsciente político de esta obra).

${ }^{7}$ La tapa y contratapa de esta obra en su primera edición jugaban con el paso entre la Galerie Vivienne, de París, y el Pasaje Güemes, de Buenos Aires. Alejandra Pizarnik (Jitrik 55), por su parte, había analizado muy tempranamente el simbolismo de las galerías en "El otro cielo" con estas palabras: "cuando el traslado sobreviene a mitad de la frase, ésta adquiere la función de una escena
} 
representado en la escisión espacial simbolizada en "El lado de acá" y "El lado de allá" de Rayuela. A la luz de estos intertextos podría verse, entonces, cada uno de los planos de "La noche boca arriba" como la lucha de un Súper-Yo en su trabajo de destrucción del Yo, a causa de un sentimiento de culpa y de la necesidad tanática de castigo. Sólo que ahora la tradicional oposición entre el Bien y el Mal de los géneros narrativos desde sus primeras manifestaciones en la cultura grecolatina, aparece extrañada por un final que podríamos llamar típicamente cortazariano como el que acontece en el relato que nos ocupa y que reitera el esquema de horror que había aparecido en otro texto como "Lejana". El hecho de que este último cuento sea anterior al viaje de su autor a Europa impide, sin embargo, entender la escisión en planos que presenta la obra posterior de Cortázar como el producto exclusivo de la alegoría del exilio y el desarraigo. De todos modos, es evidente que el giro hacia lo fantástico en las obras de Cortázar tiene que ver con un malestar hacia la excesiva importancia dada al Sujeto en la cultura occidental y cuya crítica empieza a aparecer en el posfreudismo de Lacan y el posestructuralismo francés. Esta centralización se ha revelado dentro de la misma cultura francesa como un espejismo que echa por tierra, al mismo tiempo, la omnisciencia narrativa. ¿Qué sabe ya a ciencia cierta la instancia narradora sobre el discurso que se entroniza? En realidad, muy poco (comparada con la olímpica omnisciencia balzaciana, que todavía tenía casi exclusivamente la palabra hacia 1950 en la literatura argentina). El mundo es ahora una ilusión de los sentidos. Tal vez sea Cortázar a nivel filosófico tan idealista como Borges en este momento de su producción, y el cuento del que se trata sea la arena en que sale a la lucha la forma de la expresión (como nivel genérico de la estructura narrativa) y la substancia de su expresión (los ideologemas que el relato sustenta) - para utilizar las categorías introducidas por Hjelmslev. Sea como fuere, lo cierto es que la verdadera independencia de las presiones intraculturales se manifiesta en la producción de Cortázar, cuando este autor empieza a escribir novelas, el género típicamente no borgeano, es decir en 1960 con la publicación de Los premios, y todavía más cabalmente en 1963, con la aparición de la novela Rayuela, en la que funda un nuevo género novelesco a la vez que entrega en la misma novela su poética de cómo novelar, abandonando toda idea de totalidad universal y codificación babélica. "La noche boca arriba", vista desde esta perspectiva, sería, por lo tanto, el campo de un conflicto literario que abarca toda la tradición que converge justamente en Cortázar, modelo insoslayable ahora para las generaciones posteriores.

\section{Bibliografía}

Altamirano/Sarlo. Literatura/Sociedad. Buenos Aires: Hachette, 1983. Borges, Jorge Luis. Textos cautivos. Buenos Aires: Tusquets, 1986. Obras completas 1923-1972. Buenos Aires: Emecé, 1974.

Cortázar, Julio. Obra critica. Madrid: Alfaguara, 3 tomos, 1994. "Del cuento breve y sus alrededores". Último round. México: Siglo XXI, 1969.

giratoria". La misma autora hacía alusión allí a la "traición al principio de simetría", a la vez que señalaba la percepción psicoanalítica de Cortázar como "el desdoblamiento de sí o la certidumbre (y el terror) de ser dos" (58). 
"Del sentimiento de lo fantástico". La vuelta al día en ochenta mundos. México: Siglo XXI, 1967.

Final del juego. 1956. Buenos Aires: Sudamericana, 1964.

Jameson, Fredric. The Political Unconscious. Narrative as a Socially Symbolic Act. Ithaca: Cornell University Press, 1981.

Jitrik, N. y otros autores. La vuelta a Cortázar en nueve ensayos. Buenos Aires: C. Pérez, 1969.

King, John. Sur. Estudio de la revista argentina y de su papel en el desarrollo de una cultura 1931-1970. 1986. México: Fondo de Cultura Económica, 1989.

Nash, June. "The Aztecs and the Ideology of Male Dominance". Signs,4/2 (1978).

Prieto, Adolfo. Estudios de literatura argentina. Buenos Aires: Galerna, 1969.

Soriano/Colominas. Entrevista a Cortázar bajo el título: "Lo fantástico incluye y necesita la realidad". El Pais (Madrid, 25 de marzo de 1979); reproducida en Rayuela (Edición crítica) Buenos Aires: Fondo de Cultura Económica, 1992.

Tamborenea, Mónica. Todos los fuegos, [sic] el fuego. Buenos Aires: Hachette, 1986.

Varios. The New Encyclopaedia Britannica. "Aztec"/"Pre-Columbian Civilizations". (Chicago, 33 vol., 1993). 